Research Article

\title{
Standardized Extract (HemoHIM) Protects against Scopolamine-Induced Amnesia in a Murine Model
}

\author{
Seul-Ki Kim, ${ }^{1,2}$ Da-Ae Kwon, ${ }^{2}$ Yong Sang Kim, ${ }^{3}$ Hak Sung Lee, ${ }^{4}$ Hyun Kyu Kim, \\ and Won-Ki Kim (ii) ${ }^{1}$ \\ ${ }^{1}$ Department of Neuroscience, Korea University College of Medicine, 145 Anam-ro, Seongbuk-gu, Seoul 02841, Republic of Korea \\ ${ }^{2}$ Efficacy Evaluation Team, Food Science ReD Center, Kolmar BNH Co., Ltd., 61 Heolleung-ro 8-gil, Seocho-gu, \\ Seoul, Republic of Korea \\ ${ }^{3}$ Food Safety, Food Science RßD Center, Kolmar BNH Co., Ltd., 22-15 Sandan-gil, Jeonui-myeon, \\ Sejong 30003, Republic of Korea \\ ${ }^{4}$ Natural Product Research Team, Food Science RઐD Center, Kolmar BNH Co., Ltd., 61 Heolleung-ro 8-gil, Seocho-gu, \\ Seoul, Republic of Korea \\ ${ }^{5}$ Food Science RÆD Center, Kolmar BNH Co., Ltd., 61 Heolleung-ro 8-gil, Seocho-gu, Seoul, Republic of Korea \\ Correspondence should be addressed to Won-Ki Kim; wonki@korea.ac.kr
}

Received 28 August 2020; Revised 2 March 2021; Accepted 5 March 2021; Published 17 March 2021

Academic Editor: Adolfo Andrade-Cetto

Copyright (C) 2021 Seul-Ki Kim et al. This is an open access article distributed under the Creative Commons Attribution License, which permits unrestricted use, distribution, and reproduction in any medium, provided the original work is properly cited.

\begin{abstract}
HemoHIM is a medicinal herbal preparation of Angelica gigas Nakai (Apiaceae), Cnidium officinale Makino (Umbelliferae), and Paeonia lactiflora Pallas (Paeoniaceae) designed for immune regulation. In the present study, the memory-enhancing effects of a standardized extract (HemoHIM) on scopolamine-induced memory impairment in a murine model was investigated. To induce amnesia, scopolamine $(1 \mathrm{mg} / \mathrm{kg}$ ) was intraperitoneally (i.p.) injected into mice $30 \mathrm{~min}$ before the start of behavioral tests. The Y-maze, novel object recognition test (NORT), and passive avoidance task (PAT) were used to evoke memory functions. HemoHIM significantly improved scopolamine-induced memory impairment in ICR mice, which was evidenced by an improvement of spontaneous alternation in the Y-maze, recognition index in NORT, and latency time in PAT. To elucidate the possible mechanism, the cholinergic activity and mRNA levels of choline acetyltransferase (ChAT), muscarinic acetylcholine receptor (mAchR), brain-derived neurotrophic factor (BDNF), and cAMP response element-binding protein (CREB) were measured using reverse transcription (RT-PCR) and western blot analyses, respectively. HemoHIM treatment attenuated the scopolamine-induced hyperactivation of acetylcholinesterase (AchE) activity. In addition, ChAT, mAchR, and CREB mRNA levels were increased in the hippocampus compared with the scopolamine group. Furthermore, HemoHIM treatment resulted in elevated BDNF protein expression. These results indicate that HemoHIM may exert antiamnesic activity by increasing Ach and inhibiting AchE in the hippocampus. In addition, HemoHIM has therapeutic potential by upregulating ChAT, mAchR, and BDNF, which is apparently mediated by activation of the CREB and ERK signaling pathways.
\end{abstract}

\section{Introduction}

The issue of aging is one of the most significant problems globally. Accordingly, the main problem is that social and economic costs associated with aging are increasing as the number of patients with degenerative diseases is rapidly increasing in the growing elderly population [1]. In addition, memory loss and amnesia have become important topics across all age groups in modern society. Progressive memory loss can cause serious social problems in degenerative diseases such as epilepsy, Alzheimer's disease, vascular dementia, as well as neurodegenerative and vascular disorders $[2,3]$

The hippocampus and cortex of the brain are involved in maintaining and controlling memory. Memory loss and its severity are regulated by the neurotransmitter acetylcholine (Ach), which is a chemical messenger released by nerve cells to send signals to other cells, such as neurons, muscle cells, 
and gland cells [4]. Choline uptake and Ach synthesis are decreased in the hippocampus and cerebral cortex. Conversely, the expression of acetylcholinesterase (AchE), an enzyme that degrades Ach, is activated in dementia patients [5]. Therefore, AchE inhibitors such as tacrine, donepezil, and rivastigmine have been used as therapeutic agents. However, these drugs have short half-lives and side effects, including nausea and sleep disorders [6]. Conversely, natural materials and herbs exhibit fewer adverse effects and a mixture of various ingredients has the advantage of effectively controlling complex symptoms.

Recently, various health foods using food materials and natural products have been developed around the world, and the increase in consumer demand does not match the availability of pharmaceutical products; however, immunity $[7,8]$, liver activity [9], blood circulation improvement [10], and memory improvement $[11,12]$ are areas of current public interest.

The standardized extract HemoHIM is an herbal preparation consisting of roots of Angelica gigas Nakai (Apiaceae), Cnidium officinale Makino (Umbelliferae), and Paeonia japonica Miyabe (Paeoniaceae), which reportedly inhibits various activities of human mast cells, exerts antiinflammatory effects on cigarette smoke and lipopolysaccharides, and ameliorates oxidative stress, such as stress induced by irradiation and immune-modulating activities [13-16]. HemoHIM is not a simple extract of Angelica Radix, Cnidium Rhizoma, and Paeonia Radix of Samul-Tang in oriental medicine, but rather a standardized extract of a polysaccharide fraction mixed in a certain proportion. The major components are gallic acid, chlorogenic acid, paeoniflorin, and nodakenin. We recently reported that the quantities of nodakenin, chlorogenic acid, and paeoniflorin were 106,37 , and $358 \mathrm{mg} / 100 \mathrm{~g}$, respectively, using HPLC, and we used same batch of HemoHIM in this study as well [17]. In previous studies, the herbal ingredients of HemoHIM, or their compounds, showed protective effects against amnesia and dementia [18, 19]. In particular, Angelica Radix reportedly has preventive and therapeutic effects on brain function, such as improving cognitive memory and forgetfulness, and protects against beta amyloid-induced cytotoxicity [20]. Chlorogenic acid is effective in cognitive function by increasing Ach synthesis [21-23]. With the chlorogenic acid, HemoHIM is expected to have a positive effect on cognitive enhancement.

Scopolamine is a well-known anticholinergic drug and acts by blocking the muscarinic Ach receptor (mAchR) that can interfere with memory deficits by disrupting cholinergic neurotransmission [24]. In several studies, scopolamine was reportedly used to induce amnesia in experimental models of Alzheimer's disease and has been used to screen for antiamnesic drugs [25-27]. In the present study, the memory-enhancing effects of HemoHIM in a scopolamineinduced murine model was investigated using behavioral tests, i.e., the novel object recognition test (NORT), Y-maze, and passive avoidance task (PAT). The Ach and AchE levels were subsequently investigated in the hippocampus. In addition, brain-derived neurotrophic factor (BDNF), choline acetyltransferase (ChAT), mAchRs M1 and M2, and
cAMP response element-binding protein (CREB) levels were measured using reverse transcription polymerase chain reaction (RT-PCR) analysis.

\section{Materials and Methods}

2.1. Materials. Donepezil hydrochloride monohydrate and scopolamine hydrobromide were purchased from Sigma Chemical Co. (St. Louis, MO, USA). Acetylcholine ELISA kit and acetylcholinesterase ELISA kit were purchased from Elabscience Biotechnology Co. (Houston, TX, USA). Anti$\beta$-actin antibody was purchased from Sigma Chemical Co. Anti-BDNF antibodies were purchased from Abcam (Cambridge, UK). All other materials were obtained from normal commercial sources and were of the highest grade available.

2.2. Preparation of HemoHIM. HemoHIM was prepared as described in our previous report [14]. The batch (HHH009) of standardized HemoHIM containing chlorogenic acid (25-60 mg/100 g), paeoniflorin (200-400 mg/100 g), and nodakenin (50-150 mg/100 g) was manufactured by Kolmar BNH Co., Ltd. (Sejong Si, Republic of Korea). Briefly, the traditional Korean medicinal plants, Angelica Radix (root of Angelica gigas Nakai (Apiaceae)), Cnidii Rhizoma (rhizome of Cnidium officinale Makino (Umbelliferae)), and Paeonia Radix (root of Paeonia lactiflora Pallas (Paeoniaceae)), were extracted for $4 \mathrm{~h}$ in boiling water to obtain a total extract. Half of the extract was precipitated with $95 \%$ ethanol to obtain an ethanol-insoluble polysaccharide fraction. Finally, the HemoHIM adding polysaccharide fraction was concentrated to a solid content of $30 \pm 3 \%$, freeze-dried (MCFD8512; Ilshin Lab Co., Ltd., Seoul, Republic of Korea), and stored at $4^{\circ} \mathrm{C}$ until use.

2.3. Animal. Five-week-old male ICR mice were purchased from Doo Yeol Biotech (Seoul, Korea). Animals were housed in a temperature- and humidity-controlled room at $25 \pm 2^{\circ} \mathrm{C}$ and $50 \pm 20 \%$ relative humidity, with 12-h light/12-h dark cycles. All animals had free access to food and water. All animal experiments were approved by the Korea Kolmar Animal Experimental Ethics Committee and were performed in accordance with established regulations (Approval number: $18-\mathrm{KBH}-\mathrm{S}-01)$. Animals were randomly put into five different groups $(n=13)$. Treatment administration in the five groups was as follows:

Group 1: normal saline, i.p. and p.o. (control)

Group 2: scopolamine $1 \mathrm{mg} / \mathrm{kg}$ i.p. and normal saline p.o. (negative control)

Group 3: scopolamine $1 \mathrm{mg} / \mathrm{kg}$ i.p. and donepezil $1 \mathrm{mg} /$ $\mathrm{kg}$ p.o. (positive control)

Group 4: scopolamine $1 \mathrm{mg} / \mathrm{kg}$ i.p. and HemoHIM $250 \mathrm{mg} / \mathrm{kg}$ p.o.

Group 5: scopolamine $1 \mathrm{mg} / \mathrm{kg}$ i.p. and HemoHIM $500 \mathrm{mg} / \mathrm{kg}$ p.o. 
The volume of oral (p.o.) and intraperitoneal (i.p.) administrations was $10 \mathrm{~mL} / \mathrm{kg}$ body weight of mice. For each behavioral test performed in this study, the mice were orally administered HemoHIM (250 or $500 \mathrm{mg} / \mathrm{kg}$ ) or donepezil $(1 \mathrm{mg} / \mathrm{kg}$ ) as a positive control $90 \mathrm{~min}$ before the behavioral trial (Figure 1). Memory impairment was induced by intraperitoneal (i.p.) injection of scopolamine $(1 \mathrm{mg} / \mathrm{kg})$ 30 min after the administration of HemoHIM or donepezil. The control group received normal saline.

2.4. Y-Maze Task. The Y-maze task is used as a measure of immediate spatial working memory, a form of short-term memory [28]. The Y-maze is a three-arm horizontal maze ( $40 \mathrm{~cm}$ long and $3 \mathrm{~cm}$ wide with walls $12 \mathrm{~cm}$ high) in which the three arms are symmetrically separated at $120^{\circ}$. The apparatus floor and walls were constructed from black polyvinyl plastic. Mice were initially placed in one arm, and the arm entry sequence (e.g., $\mathrm{ABCCAB}$, where letters indicate arm-codes) and the number of arm entries were recorded manually for each mouse over an 8-min period. An actual alternation was defined as entries into all three arms consecutively (i.e., $\mathrm{ABC}, \mathrm{CAB}$, or $\mathrm{BCA}$ but not $\mathrm{BAB}$ ). Mice were orally administered HemoHIM (250 or $500 \mathrm{mg} / \mathrm{kg}$ ) or donepezil $(1 \mathrm{mg} / \mathrm{kg}) \quad 1.5 \mathrm{~h}$ before the acquisition trial. Memory impairment was induced by scopolamine treatment ( $1 \mathrm{mg} / \mathrm{kg}$, i.p.) $30 \mathrm{~min}$ after the administration of HemoHIM, donepezil, or normal saline. Maze arms were thoroughly cleaned between tests to remove residual odors. Percentage alternation was determined by dividing the total number of alternations by the total number of choices minus 2 multiplied by 100 as shown by the following equation:

Alternation $(\%)=($ Number of alternations/Total arm entries -2$) \times 100$. The number of arm entries served as an indicator of locomotor activity. The number of total arm entries was measured as an indicator for animal behavior activity.

2.5. Novel Object Recognition Test. To assess an object recognition memory, the NORT was performed as previously described [29]. The NORT test was conducted 1 day after the Y-maze test. Mice were orally administered HemoHIM (250 or $500 \mathrm{mg} / \mathrm{kg})$ or donepezil $(1 \mathrm{mg} / \mathrm{kg}) 1 \frac{1}{2} h$ before NORT. Memory impairment was induced by scopolamine treatment $(1 \mathrm{mg} / \mathrm{kg}$, i.p.) $30 \mathrm{~min}$ after administration of HemoHIM or donepezil. The experimental apparatus consisted of a polyvinyl plastic square open field $(25 \mathrm{~cm} \times 25 \mathrm{~cm} \times 25 \mathrm{~cm})$. Habituation training was conducted by exposing the animal to the experimental apparatus for 5 min per day in the absence of objects. During the test, mice were placed in the experimental apparatus in the presence of two identical objects and allowed to explore for $5 \mathrm{~min}$. After a retention interval of $24 \mathrm{~h}$, mice were again placed in the apparatus; however, one of the objects was replaced with a novel object. Mice were also allowed to explore for $5 \mathrm{~min}$. The objects chosen for this experiment were approximately of the same height. The duration of time mice spent exploring each object (the number of exploring familiar object, N1; the number of exploring the novel object, N2) was recorded. The recognition index $(\%)=\mathrm{N} 2 /(\mathrm{N} 2+\mathrm{N} 1) \times 100$.

2.6. Passive Avoidance Task. The PAT was conducted in identical illuminated and nonilluminated boxes (Avoidance System, version 1.1; BS Techno-lab Inc., Seoul, Korea). The test is an automatic learning method designed for active and passive avoidance experiments in animals. The test station consists of two chambers, and the bottom consists of a grid that can cause an electric shock. These two compartments were separated by a guillotine door $(5 \times 5 \mathrm{~cm})$. For the acquisition trial, mice were initially placed in the illuminated compartment and the door between the two compartments was opened $10 \mathrm{sec}$ later. When mice entered the dark compartment, the door automatically closed and an electrical foot shock $(0.3 \mathrm{~mA}) 3 \mathrm{sec}$ in duration was delivered through the stainless steel rods. Mice were again placed in the illuminated compartment $24 \mathrm{~h}$ after the acquisition trial to test retention. The time measured for a mouse to enter the dark compartment after the door opened was defined as latency up to $300 \mathrm{sec}$. The test was conducted as previously described with modifications [30].

2.7. RNA Isolation and RT-PCR. Total RNA was extracted from the hippocampus of ICR mice using the RNeasy Mini Kit (Qiagen, Hilden, Germany) and quantified with a NanoDrop 2000 UV-Vis spectrophotometer (Thermo Fisher Scientific Inc., Waltham, MA, USA). cDNA was synthesized from extracted total RNA using a High-Capacity cDNA Reverse Transcription Kit (Applied Biosystems, Carlsbad, CA, USA). The cDNA of genes of interest was amplified using AccuPower PCR Premix (Bioneer, Daejeon, Korea). Table 1 shows the primer sequences used for RT-PCR. The amplified cDNA was electrophoresed on 1.8\% agarose gel and stained with ethidium bromide (EtBr). The expression level of the target mRNA was measured using $\beta$-actin as a control and analyzed with Image J software (NIH, Framingham, MA, USA).

2.8. Biochemical Assay. Briefly, mice were sacrificed by decapitation and the brain was immediately removed to isolate the cortex or hippocampus. A hippocampus tissue was added to a microfuge tube containing cold phosphatebuffered saline (PBS) and homogenized using a homogenizer. The resulting tissue was then centrifuged at $13,000 \mathrm{rpm}$ and $4^{\circ} \mathrm{C}$. AchE and Ach levels in the hippocampus were determined using the acetylcholine ELISA kit and acetylcholinesterase ELISA kit (Elabscience Biotechnology Co.) according to the manufacturer's instructions.

2.9. Western Blotting. Isolated tissues were homogenized in ice-chilled Tris- $\mathrm{HCl}$ buffer ( $20 \mathrm{mM}$, pH 7.4). Homogenate samples $(15 \mu \mathrm{g}$ of protein) were subjected to SDS-PAGE under reducing conditions. Proteins were transferred onto $20 \mu \mathrm{m}$ PVDF membranes in transfer buffer and further separated at $100 \mathrm{~V}$ for $2 \mathrm{~h}$ at $4^{\circ} \mathrm{C}$ to determine expression levels. The western blots were incubated for $2 \mathrm{~h}$ with 


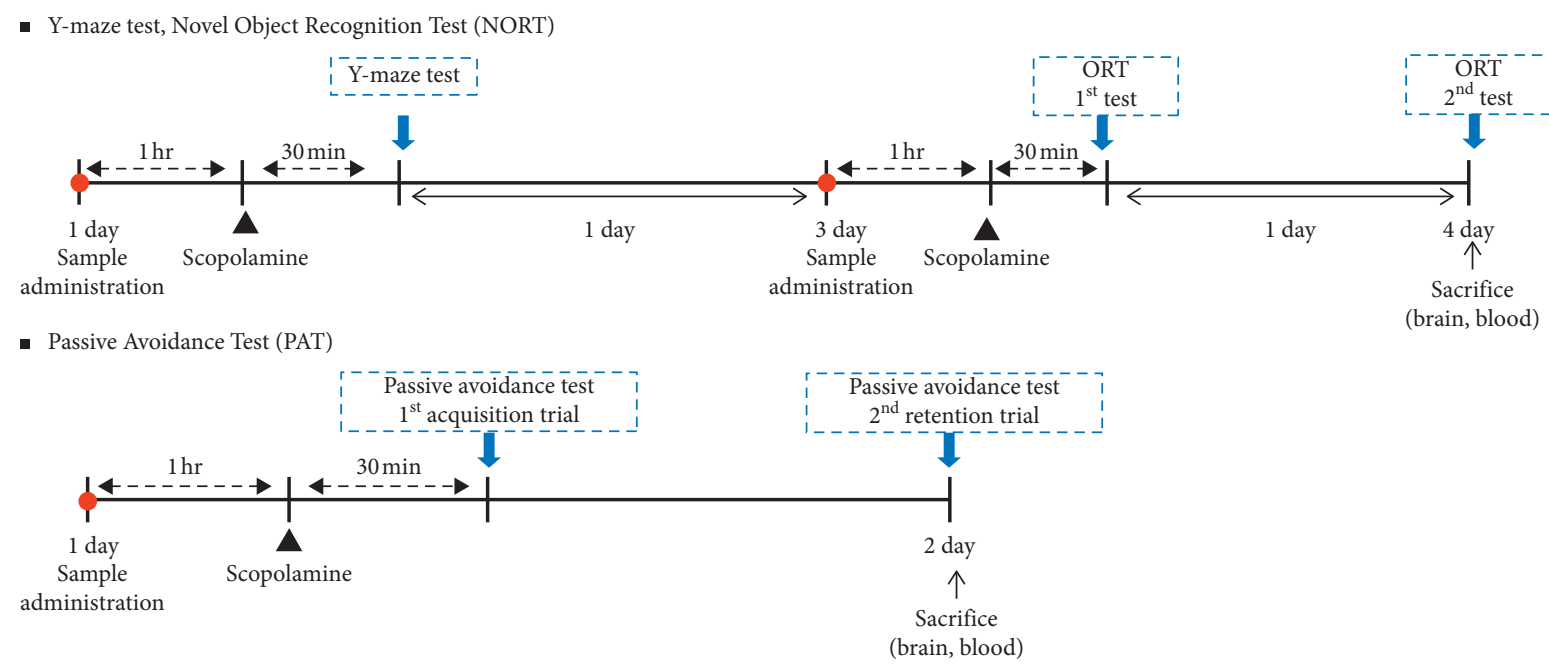

FIGURE 1: Schematic description of the behavioral experimental design. ICR mice were orally treated with vehicle, HemoHIM $250,500 \mathrm{mg} /$ $\mathrm{kg}$, or donepezil $1 \mathrm{mg} / \mathrm{kg}$ before the behavioral experiment. Animals were randomly put into five different groups ( $n=13)$. Scopolamine $1 \mathrm{mg} / \mathrm{kg}$ was i.p. injected $1 \mathrm{~h}$ after sample administration. The behavioral test was performed $30 \mathrm{~min}$ after scopolamine injection.

TABLE 1: Primer sequences used for reverse transcriptase PCR.

\begin{tabular}{lcc}
\hline Gene & Direction & Sequence $\left(5^{\prime}\right.$ to $\left.3^{\prime}\right)$ \\
\hline \multirow{2}{*}{ AchE } & Forward & AGAAAATATTGCAGCCTTTG \\
& Reverse & CTGCAGGTCTTGAAAATCTC \\
ChAT & Forward & AGGGTGATCTGTTCACTCAG \\
& Reverse & TCTTGTTGCTGTCATCATA \\
M1 AchR & Forward & CAGAAGTGGAGATGCC \\
& Reverse & GAGCTTTTGGGAGGCTGCTT \\
M2 AchR & Forward & TGCTGTGGCCTCCAATATGA \\
& Reverse & TGACCCGACGACCCAACT \\
BDNF & Forward & AGCTGAGCGTGTGTGACAGTAT \\
& Reverse & CCGAACATACGATTGGGTAGTT \\
Trk $\beta$ & Forward & GCACATCGCTCAGCAAATCG \\
& Reverse & ACAACTCCCAGGCTCCAGAC \\
CREB & Forward & CCCAGGGAGGAGCAATACAG \\
& Reverse & GGGAGGACGCCATAACAACT \\
ERK1 & Forward & ACCGTGACCTCAAGCCTTCC \\
& Reverse & GATGCAGCCCACAGACCAAA \\
ERK2 & Forward & TTGCTGAAGCACCATTCAAG \\
& Reverse & ACGGCTCAAAGGAGTCAAGA \\
$\beta$-actin & Forward & GCCATGTACGTAGCCATCCA \\
& Reverse & GAACCGCTCATTGCCGATAG \\
\hline
\end{tabular}

blocking solution ( $5 \%$ skim milk) at $4^{\circ} \mathrm{C}$ followed by overnight incubation with primary antibodies. The blots were then washed twice with Tween 20/Tris-buffered saline (TTBS), incubated with a 1:5,000 dilution of horseradish peroxidase-conjugated secondary antibody for $1 \mathrm{~h}$ at room temperature, washed three times with TTBS, and developed using enhanced chemiluminescence (Amersham Life Science, Arlington Heights, IL, USA). Immunoblots were imaged using the bioimaging program on a LAS-4000 mini imager (Fujifilm Lifescience USA, Stamford, CT, USA) and analyzed using Multi Gauge version 3.2 (Fujifilm Holdings Corporation, Tokyo, Japan). The BDNF level was determined by calculating the ratios of BDNF to the corresponding $\beta$-actin on the same membranes.
2.10. Statistical Analysis. All results are presented as the mean \pm standard error of the mean (S.E.M). One-way analysis of variance (ANOVA) followed by Duncan's multiple range test were used for comparing three or more groups. The GraphPad Prism5.0 (GraphPad Prism Software Inc., San Diego, CA, USA) program was used for statistical analysis. $P$ values $<0.05$ were considered statistically significant.

\section{Results}

3.1. Effects of HemoHIM on Learning and Memory Improvement in Behavioral Tests. All experimental groups showed normal results regarding general behavioral changes, and adverse events were not observed. In addition, body weight and food intake measured during treatment in all animal groups were not significantly different among the groups (data not shown).

The Y-maze test was performed to assess whether HemoHIM improved memory impairment induced by scopolamine. As shown in Figure 2(a), spontaneous alternation in the scopolamine-treated group was significantly decreased compared with the control group $(21.50 \pm 5.07 \%$ and $56.67 \pm 4.01 \%$, respectively) $(P<0.05)$. Furthermore, HemoHIM significantly reversed the reduced alternation induced by scopolamine at $250 \mathrm{mg}(47.38 \pm 5.11 \%)$ and $500 \mathrm{mg} / \mathrm{kg}(56.41 \pm 4.57 \%)$. In addition, the effects of HemoHIM on spontaneous alternation were similar to that of donepezil $(53.27 \pm 3.56 \%)$. However, the number of total arm entry had not significantly changed compared with each experimental group (Figure 2(b)). Therefore, it was confirmed that HemoHIM or donepezil did not affect mice locomotor activity.

The NORT is a commonly used behavioral test for the investigation of various aspects of learning and memory in rodents. Mice in the scopolamine-treated group spent significantly longer time exploring the familiar object than exploring the novel object (Figure 3(b)). The percentage 


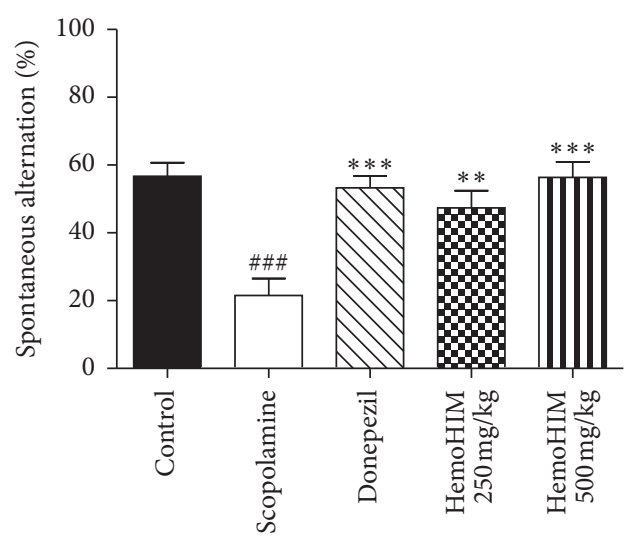

(a)

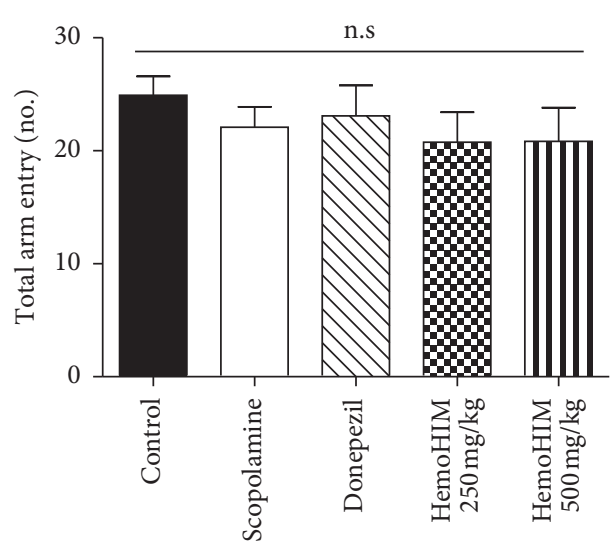

(b)

Figure 2: The effects of HemoHIM on scopolamine-induced memory impairment in the Y-maze test. Spontaneous alternation (\%) was calculated as follows. $\%=($ Number of alternations/Total arm entries -2$) \times 100$. Spontaneous alternation $(\mathrm{a})$ and numbers of total arm entry (b) were measured for Y-maze test. Ninety minutes before Y-maze, mice were treated with HemoHIM (250 and $500 \mathrm{mg} / \mathrm{kg}$, p.o.) or donepezil (1 mg/kg, p.o.) as a positive control. Memory impairment was induced by scopolamine treatment (1 mg/kg, i.p.). Thirteen different animals were used per group. The data are expressed as the mean \pm S.E.M. Significant difference from control group $(\# \# \# P<0.001)$ and from scopolamine-treated group $\left({ }^{* * *} P<0.001,{ }^{* *} P<0.01\right)$.

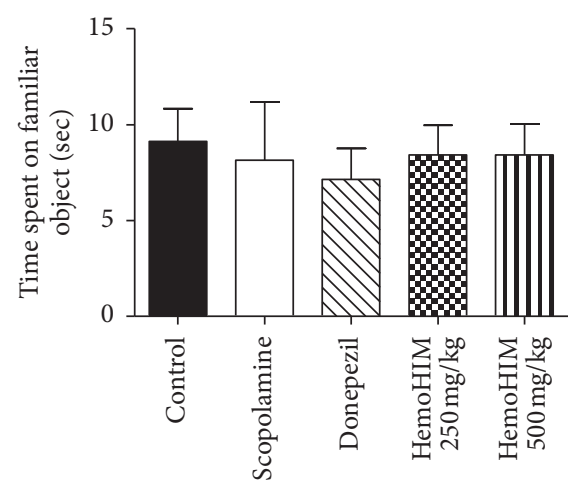

(a)

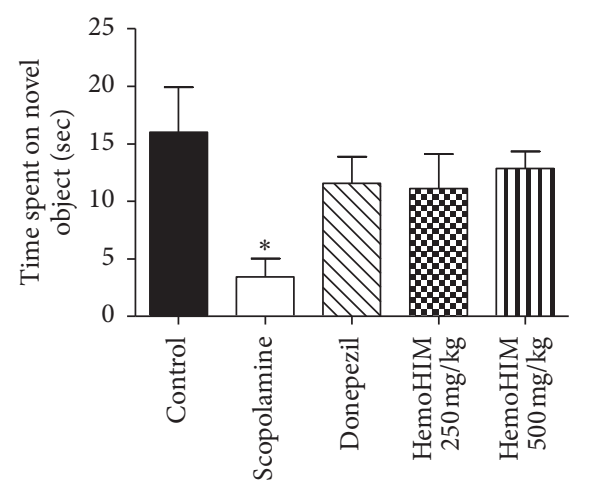

(b)

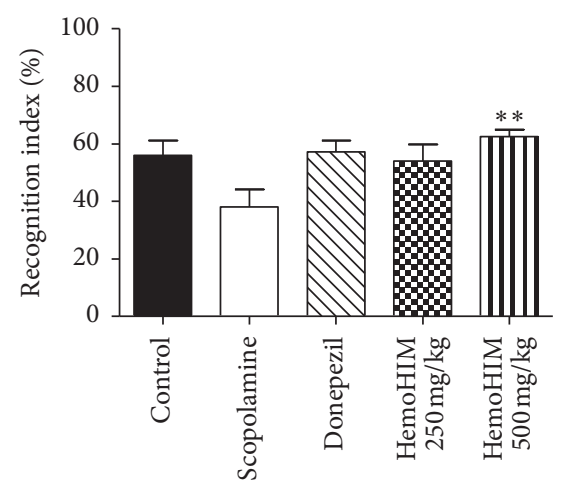

(c)

Figure 3: The effects of HemoHIM on scopolamine-induced memory impairment in the NORT. (a) Time spent on familiar object (sec), (b) time spent novel object (sec), and (c) Recognition index (\%). Ninety minutes before Y-maze, mice were treated with HemoHIM (250 and $500 \mathrm{mg} / \mathrm{kg}$, p.o.) or donepezil (1 mg/kg, p.o.) as a positive control. Memory impairment was induced by scopolamine treatment (1 mg/kg, i.p.). Thirteen different animals were used per group. The data are expressed as the mean \pm S.E.M. Significant difference from control group $(\# P<0.05)$ and from scopolamine-treated group $\left({ }^{* *} P<0.01\right)$.

recognition index was not increased in the positive control and HemoHIM $250 \mathrm{mg} / \mathrm{kg}$ group $(57.17 \pm 4.01 \%$ and $54.01 \pm 5.83 \%$, respectively). However, the recognition index in the HemoHIM $500 \mathrm{mg} / \mathrm{kg}$ group was notably higher than that in the scopolamine-treated group $(62.65 \pm 2.37 \%$ and $38.02 \pm 6.15 \%$, respectively, Figure $3(\mathrm{c}))$. Mice in the HemoHIM $500 \mathrm{mg} / \mathrm{kg}$ group spent more time exploring the novel object than exploring the familiar object. Therefore, the high concentration of HemoHIM was confirmed to improve cognitive impairment.

The effects of HemoHIM on long-term memory were investigated using the PAT (Figure 4). The results showed the time delay to enter into the dark room was significantly reduced in the scopolamine-treated group compared with the control group $(111.07 \pm 59.81 \mathrm{sec}$ and $61.6 \pm 7.96 \mathrm{sec}$, respectively, Figure 4). Conversely, donepezil $(162.06 \pm 31.19 \mathrm{sec})$ and HemoHIM $(500 \mathrm{mg} / \mathrm{kg})$ significantly increased the time latency $(171.10 \pm 30.44 \sec P<0.01$ and $P<0.05$, respectively).

3.2. Effects of HemoHIM on Cholinergic Activity in the Hippocampus. In previous studies, several natural compounds or extracts with AchE inhibitory activity were reported to exhibit significant cognitive improvement effects. Therefore, hippocampal Ach and AchE activities were measured in the present study to determine the fundamental mechanism underlying the possible alteration of Ach and AchE levels. The activity of Ach slightly decreased in the scopolamine-treated group $(2.61 \pm 0.25)$ compared with 


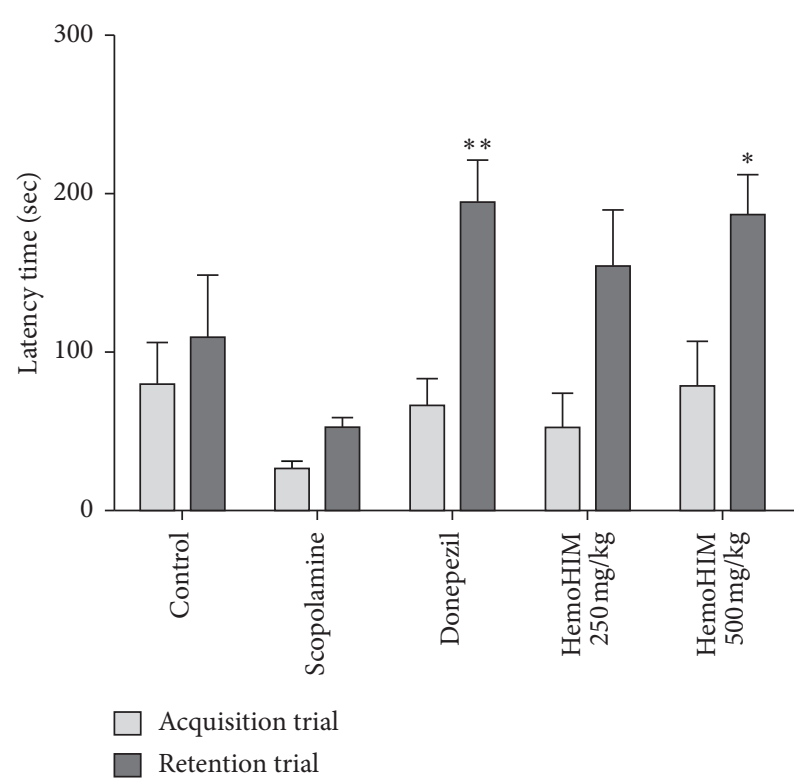

Figure 4: Effects of HemoHIM (250 and $500 \mathrm{mg} / \mathrm{kg}$, p.o.) on scopolamine-induced memory impairment in the passive avoidance test in mice. The results from the acquisition trial and retention trial were presented. Ninety minutes before Y-maze, mice were treated with HemoHIM (250 and $500 \mathrm{mg} / \mathrm{kg}$, p.o.) or donepezil ( $1 \mathrm{mg} / \mathrm{kg}$, p.o.) as a positive control. Memory impairment was induced by scopolamine treatment $(1 \mathrm{mg} / \mathrm{kg}$, i.p.). Thirteen different animals were used per group. The data are expressed as the mean \pm S.E.M. Significant difference from scopolamine-treated group $\left({ }^{* *} P<0.01,{ }^{*} P<0.05\right)$.

controls $(3.05 \pm 0.06)$. However, donepezil and HemoHIM $500 \mathrm{mg} / \mathrm{kg}$ groups showed a significant increase in Ach levels $(3.39 \pm 0.05$ and $3.11 \pm 0.11$, respectively, Figure 5(a)). As shown in Figure 5(b), the scopolamine-increased activity of AchE was significantly reduced by the administration of HemoHIM $(500 \mathrm{mg} / \mathrm{kg})$ and by the donepezil treatment $(P<0.05$ and $P<0.001$, respectively).

\subsection{Effects of HemoHIM on the mRNA Levels of Ach-Related} Metabolic Enzymes and Receptors. The effects of HemoHIM on cognitive memory enhancement were investigated. The Ach-related metabolic enzymes and receptors were analyzed using RT-PCR. As shown in Figure 6(a), the ChAT activity decreased more in the scopolamine-treated group than in controls; however, the HemoHIM $500 \mathrm{mg} / \mathrm{kg}$ group showed significantly increased ChAT activity compared with the scopolamine-treated group. The mAchR (M1 and M2) mRNA expression was significantly lower in the scopolamine-treated group than in controls (Figures 6(c), 6(d)). However, HemoHIM strongly increased M1 and M2 expression in a dose-dependent manner. Scopolamine administration notably upregulated AchE expression in the hippocampus. Administration of HemoHIM and donepezil induced a significant attenuation of AchE expression (Figure 6(b)). Scopolamine treatment not only reduced BDNF but also decreased the amount of ERK and CREB. Conversely, the HemoHIM groups showed significantly increased ERK, BDNF, and CREB expressions (Figures 6(e), $6(\mathrm{~g})-6(\mathrm{i}))$.

3.4. Effects of HemoHIM on the Expression of BDNF Activation. To examine whether HemoHIM influences protein expression associated with long-term memory, western blotting for BDNF in hippocampal tissue was performed (Figure 7). Treatment with HemoHIM $(500 \mathrm{mg} /$ $\mathrm{kg}$ ) significantly upregulated BDNF expression in the hippocampus. The positive control also showed significantly increased BDNF expression. The result indicates HemoHIM may protect against scopolamine-induced amnesia via mechanisms associated with neuronal cell activation.

\section{Discussion}

The herbal preparation HemoHIM is a functional health food, which has obtained certification by the Ministry of Food and Drug Safety (MFDS) to improve immune function. HemoHIM consists of hot water extract with a polysaccharide fraction of herbs, Angelica gigas Nakai (Apiaceae), Cnidium officinale Makino (Umbelliferae), and Paeonia japonica Miyabe (Paeoniaceae). Various effects of immune enhancement in HemoHIM such as immune cell activation, immune hematopoietic recovery, tissue regeneration, and antioxidant effects have been reported $[7,9,11,12]$. The major components of HemoHIM are gallic acid, chlorogenic acid, paeoniflorin, and nodakenin. In previous studies, Kim et al. and Kwon et al. reported that nodakenin and chlorogenic acid might be useful for the treatment of cognitive impairment via the antioxidant activity and the enhancement of cholinergic signaling $[18,21]$.

In the present study, the memory-enhancing effects of HemoHIM were investigated in a scopolamine-induced mouse model of amnesia using behavioral tests and the related mechanisms. Scopolamine, a muscarinic antagonist, can cause learning and memory deficits by disrupting cholinergic activity. Consequently, scopolamine has often been widely used to screen potential agents as effective treatment for antiamnesia or dementia in experimental murine models $[18,27,31,32]$. In the present investigation, the learning and memory function were evaluated in experimental animals using the Y-maze, NORT, and PAT. These behavioral tests are useful for studying short- and long-term memory by manipulating the retention interval $[30,33]$. In previous studies, scopolamine-treated animals showed a lower discrimination index in the NORT, a higher transfer latency in the elevated plus-maze test, and a longer latency in the Morris water maze test $[34,35]$. Increasing spontaneous alternation indicates that learning and memory have been restored. In the Y-maze task, HemoHIM administration $(250$ or $500 \mathrm{mg} / \mathrm{kg})$ significantly increased spontaneous alternation behavior in mice, ameliorating the decreased alternation behavior that was induced by scopolamine. Furthermore, this was similar to the effects of donepezil. The NORT is universally recognized for short- or long-term memory. In the present study, scopolaminetreated mice exhibited significantly decreased exploration 


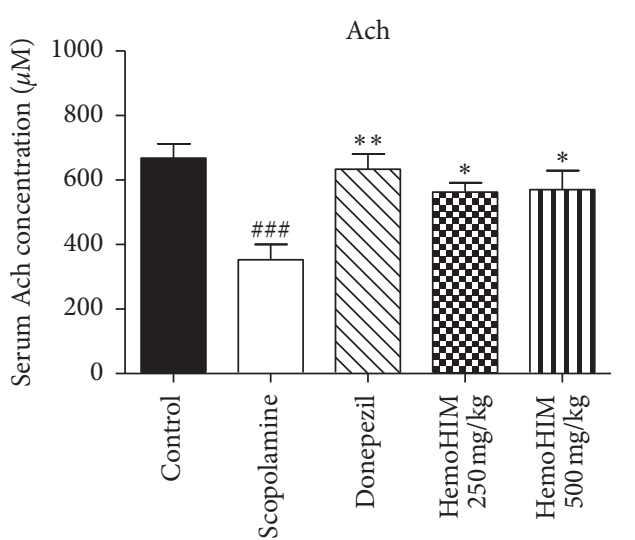

(a)

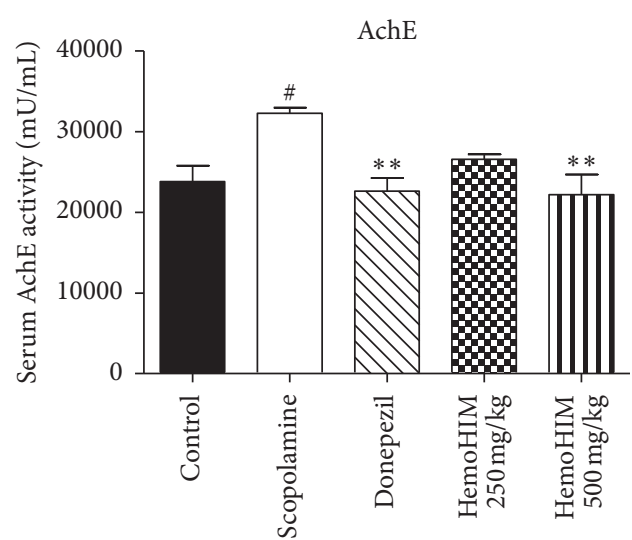

(b)

Figure 5: The effects of HemoHIM on (a) Ach concentration and (b) AchE activity in the hippocampus. Ach levels ( $n=$ five per group) and AchE ( $n=6$ per group) in the hippocampus were determined using the ELISA kit. The data are expressed as the mean \pm S.E.M. Significant difference from control group (\#\#\#< $<0.001)$ and from scopolamine-treated group $\left({ }^{* * *} P<0.001,{ }^{*} P<0.05\right)$.

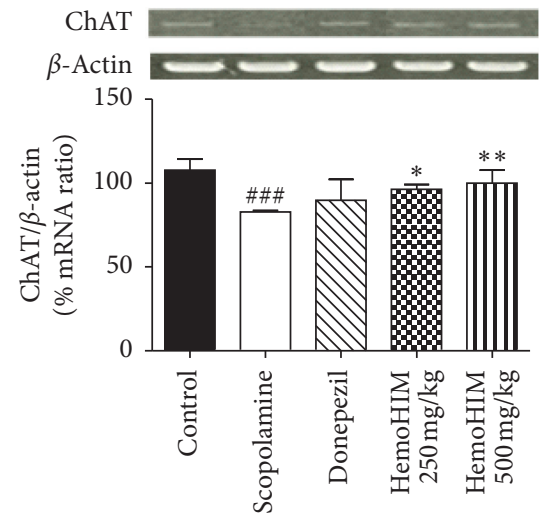

(a)

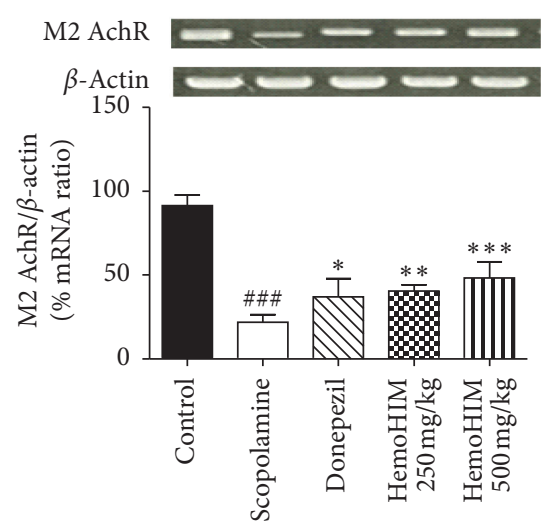

(d)

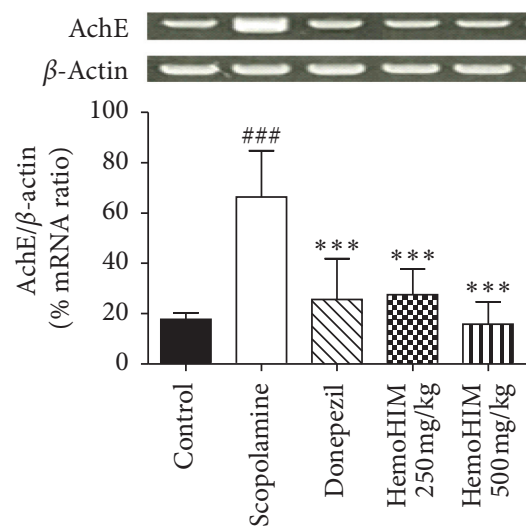

(b)

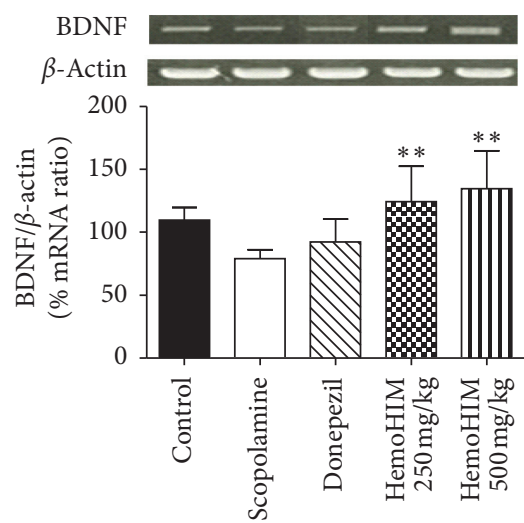

(e)

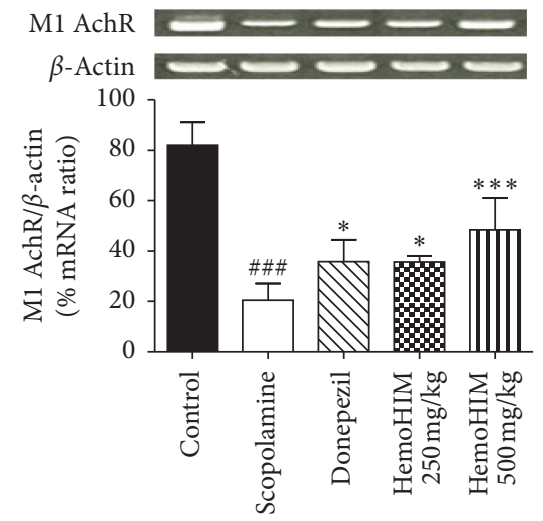

(c)

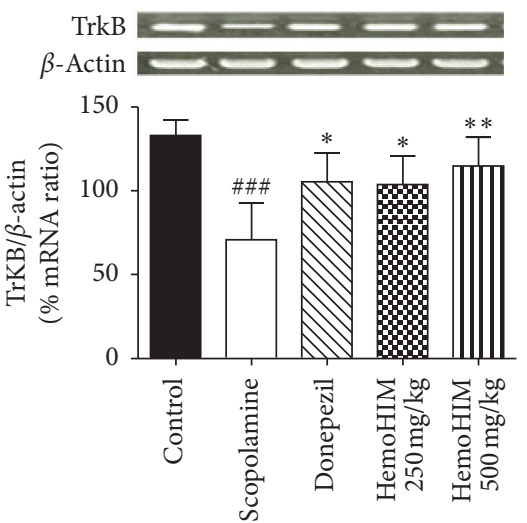

(f)

Figure 6: Continued. 


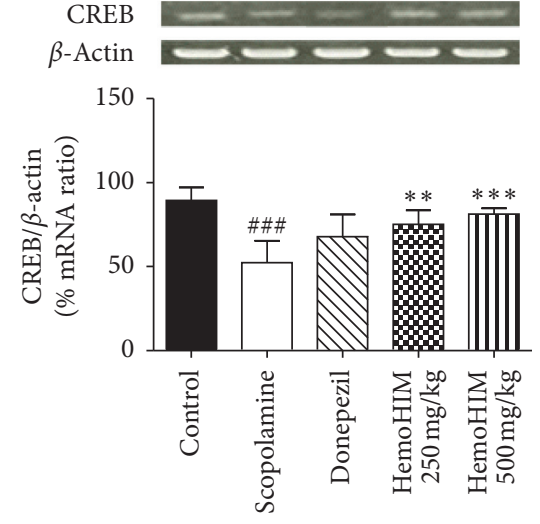

(g)
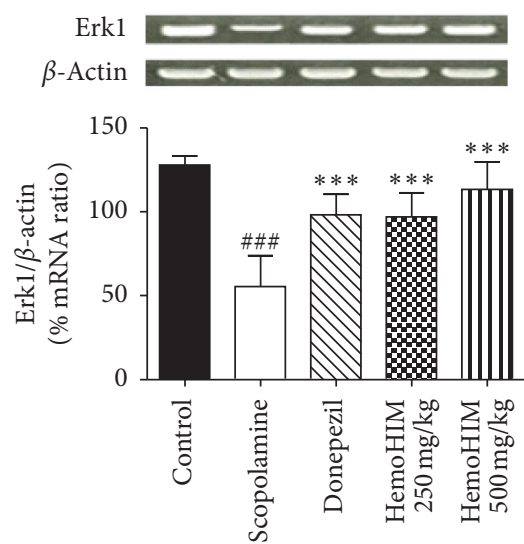

(h)

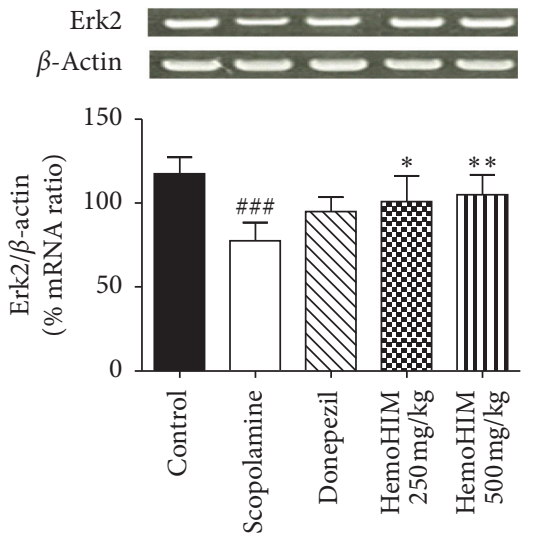

(i)

Figure 6: The effects of HemoHIM on mRNA expression of (a) ChAT, (b) AchE, (c) mAchR M1, (d) mAchR M2, (e) BDNF, (f) TrkB, (g) CREB, (h) Erk1, and (i) Erk2 in the scopolamine-induced model. The mRNA expression was measured by RT-PCR using specific primers. $\beta$-actin levels were compared for the equal loading control. Quantitative analysis of relative expression for BDNF/ $\beta$-actin was represented as graph. The data are expressed as the mean \pm S.E.M. ( $n=4 \sim 5$ per group). Significant difference from control group $(\# \# P<0.001)$ and from scopolamine-treated group $\left({ }^{* * *} P<0.001,{ }^{* *} P<0.01,{ }^{*} P<0.05\right)$.

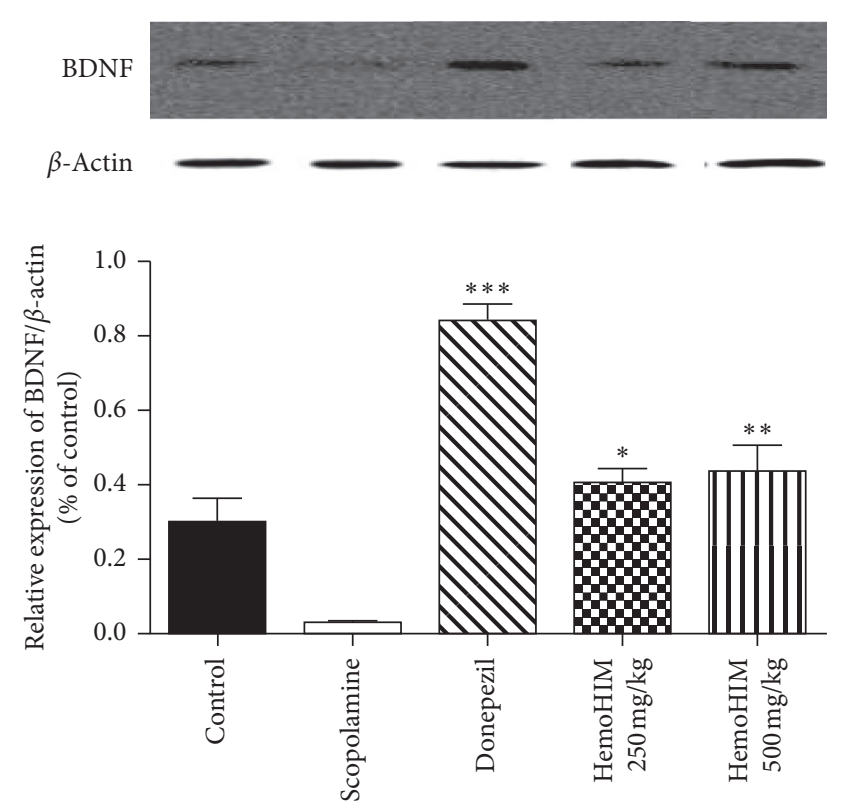

Figure 7: The effects of HemoHIM on BDNF protein levels in the hippocampus. Protein expression was measured by western blot analysis using anti-BDNF-specific antibody. $\beta$-actin levels were compared for the confirmation of an equal amount of protein loading in each group. Quantitative analysis of relative expression for $\mathrm{BDNF} / \beta$-actin was represented as graph. The data are expressed as the mean \pm S.E.M. ${ }^{* * *} P<0.001,{ }^{* *} P<0.01$, and ${ }^{*} P<0.05$ compared with the scopolamine-treated group.

time showing a novelty preference, indicating that scopolamine causes impairment of object recognition memory. In contrast, mice administered HemoHIM (500 mg/kg) showed novelty preference by spending more time on the novel object relative to the familiar object. The PAT is a fearmotivation test that is classically used to assess short- or long-term memory using the natural tendency of rodents for dark places [36]. The amount of time a mouse stays in the brightly lit box before entering the dark box, where an electric shock is administered, is considered the latency time. Longer latency time indicates improvement of learning and memory function. In the present study, latency time was compared and difference was not observed between groups in the acquisition trial. However, the latency time was reduced in the scopolamine-treated group in the retention trial. The results showed that HemoHIM treatment improved latency time during trials, indicating the amelioration of learning impairment.

Dementia, which appears to be the cause of aging and Alzheimer's disease, is a neurodegenerative brain disease and considered an important social problem as well as a major disease in modern society [37]. Memory impairment is the earliest and the most prominent symptom of dementia. Memory is related to the cerebral cortex, especially the hippocampus. The cholinergic deficits are neuropathological occurrences that are consistently associated with memory impairment in the hippocampus and play an important role in memory formation and cognition $[38,39]$. In addition, reduction in memory function is correlated with dementia. In several reports, choline uptake and Ach synthesis were reduced in patients with dementia [40, 41]. In addition, many approaches lead to enhancing the concentration of acetylcholine. Many studies have been conducted to increase the content of endogenous Ach by blocking the action of AchE, which hydrolyzes Ach. As in previous studies, the administration of scopolamine reduced Ach concentration and elevated AchE activity in the present study [27]. On the other hand, HemoHIM was able to confirm Ach significant increase and AchE activity inhibition in the serum and hippocampus (Figures 3, 4). Conversely, HemoHIM significantly increased Ach and inhibited AchE activity in the serum and hippocampus (Figure 3, 4). HemoHIM increased the Ach level similar to donepezil, which was used as a control drug. These findings indicate that HemoHIM decreases the activity of AchE, which hydrolyzes Ach, 
consequently increasing the endogenous Ach and improving cholinergic neurotransmission.

To investigate the effects of HemoHIM, the expression of receptors that can influence the synthesis, release, and degradation of Ach, a neurotransmitter that plays a central role in memory and learning, was investigated. ChAT, a transferase enzyme responsible for the synthesis of the Ach, is important for performing basic brain functions. mAchRs are Ach receptors that form G protein-coupled receptor complexes in the cell membranes. mAchRs M1 and M2 are the most abundant muscarinic receptors in the hippocampus and were shown to exert cognitive effects [42], and M2deficient mice had disrupted hippocampal Ach homeostasis [43]. Decreased ChAT and mAchR activity, especially in the hippocampus, has been previously reported in patients with dementia [44]. In the present study, reduced ChAT and mAchRs M1 and M2 levels were observed in the scopolamine-treated group; however, the expression levels were increased in the HemoHIM $500 \mathrm{mg} / \mathrm{kg}$ group. Thus, based on the findings from the present and previous studies, HemoHIM likely improves Ach synthesis by increasing ChAT and mAchRs M1 M2 levels and decreasing AchE activity. In addition, expression of transcription and neurotrophic factors such as CREB and BDNF was observed. BDNF binds to its receptor TrkB and activates tyrosine kinase, a trigger for various intercellular signaling pathways [45]. The BDNF and TrkB expression was significantly increased in the HemoHIM groups. These neurotrophic factors are being developed as biomarkers and new drug targets for mental nervous system diseases such as memory impairment and depression and/or anxiety. CREB is one of the key signaling molecules involved in learning and retrieval of fear-based long-term memory and are activated by phosphorylation on serine 133, which can be controlled by ERK signaling [46]. Especially, BDNF is a member of the neurotrophin family of growth factors and appears essential for molecular mechanisms of synaptic transmission and synapse plasticity in the central nervous system [47]. Our result is HemoHIM $(500 \mathrm{mg} / \mathrm{kg})$ treatment significantly upregulated BDNF expression in the hippocampus. Therefore, HemoHIM high dose can increase BDNF expression and help improve cognitive memory. However, further studies are needed to identify the molecular biological mechanism of action and signal pathways other than BDNF.

\section{Conclusion}

The present study was conducted to evaluate the antiamnesic activity of HemoHIM in a scopolamine-induced memory impairment murine model. HemoHIM administration effectively ameliorated cognitive deficits determined using PAT, NORT, and Y-maze behavioral tests. HemoHIM effectively inhibited AchE activity. Furthermore, HemoHIM may attenuate memory impairment by activating the BDNFERK-CREB pathway. These results indicate that HemoHIM can be used pharmaceutically for the treatment or prevention of amnesia and dementia and can be used as a supplement to improve dementia symptoms.

\section{Data Availability}

The data used to support the findings of this study are available from the corresponding author upon request.

\section{Conflicts of Interest}

The authors declare that there are no conflicts of interest.

\section{Authors' Contributions}

All authors contributed to this study. W.K. Kim and H.K. Kim conceived and designed the experiments; S.K. Kim, D.A. Kwon, Y.S. Kim, and H.S. Lee performed the experiments; S.K. Kim, D.A. Kwon, Y.S. Kim, and H.S. Lee contributed to the interpretation and statistical analysis of the data; and S.K. Kim wrote the manuscript.

\section{Acknowledgments}

This research was supported by a grant from Kolmar BNH, Korea.

\section{References}

[1] A. Wimo, L. Jönsson, J. Bond, M. Prince, B. Winblad, and A. D. International, "The worldwide economic impact of dementia 2010," Alzheimer's \& Dementia, vol. 9, no. 1, pp. 1-11, e13, 2013.

[2] J. Prabhu, K. Prabhu, A. Chaudhuri et al., "Neuro-protective effect of ayurveda formulation, saraswatharishtam, on scopolamine induced memory impairment in animal model," Pharmacognosy Journal, vol. 12, no. 1, 2020.

[3] S. J. Lee, T. H. Kim, L. Huh et al., "The correlation of levels of serum lipid, homocysteine, and folate with volumes of Hippocampus, amygdala, corpus callosum, and thickness of entorhinal cortex in patients with amnestic mild cognitive impairment or dementia of Alzheimer's type," Korean Journal of Biological Psychiatry, vol. 22, no. 4, p. 223, 2015.

[4] M. E. Hasselmo, "The role of acetylcholine in learning and memory," Current Opinion in Neurobiology, vol. 16, no. 6, pp. 710-715, 2006.

[5] B. Hassel, V. Solyga, and A. Lossius, "High-affinity choline uptake and acetylcholine-metabolizing enzymes in CNS white matter. A quantitative study," Neurochemistry International, vol. 53, no. 6-8, pp. 193-198, 2008.

[6] D. W. Lee, Y. S. Huh, and K. W. Kim, "Evidence-based treatment of Alzheimer's disease," Journal of the Korean Medical Association, vol. 52, no. 4, pp. 417-425, 2009.

[7] E. M. Noh, J. M. Kim, H. Y. Lee et al., "Immuno-enhancement effects of Platycodon grandiflorum extracts in splenocytes and a cyclophosphamide-induced immunosuppressed rat model," BMC Complementary and Alternative Medicine, vol. 19, no. 1, pp. 1-12, 2019.

[8] K. Wang, H. Zhang, Q. Han et al., "Effects of astragalus and ginseng polysaccharides on growth performance, immune function and intestinal barrier in weaned piglets challenged with lipopolysaccharide," Journal of Animal Physiology and Animal Nutrition, vol. 104, 2020.

[9] T. C. Peixoto, E. G. Moura, E. de Oliveira et al., "Cranberry (Vaccinium macrocarpon) extract treatment improves triglyceridemia, liver cholesterol, liver steatosis, oxidative damage and corticosteronemia in rats rendered obese by high 
fat diet," European Journal of Nutrition, vol. 57, no. 5, pp. 1829-1844, 2018.

[10] J. Lee, G. Kwon, J. Park et al., "An ethanol extract of Ramulus mori improves blood circulation by inhibiting platelet aggregation," Bioscience, Biotechnology, and Biochemistry, vol. 80, no. 7, pp. 1410-1415, 2016.

[11] R. Krikorian, W. Kalt, J. E. McDonald, M. D. Shidler, S. S. Summer, and A. L. Stein, "Cognitive performance in relation to urinary anthocyanins and their flavonoid-based products following blueberry supplementation in older adults at risk for dementia," Journal of Functional Foods, vol. 64, p. $103667,2020$.

[12] W. Y. Choi, D. H. Kang, and H. Y. Lee, "Effect of fermented Spirulina maxima extract on cognitive-enhancing activities in mice with scopolamine-induced dementia," Evidence-Based Complementary and Alternative Medicine, vol. 2018, Article ID 7218504, 9 pages, 2018.

[13] S.-K. Kim, D. Kwon, H. S. Lee, H. K. Kim, and W.-K. Kim, "Preventive effect of the herbal preparation, HemoHIM, on cisplatin-induced immune suppression," Evidence-Based Complementary and Alternative Medicine, vol. 2019, Article ID 3494806, 8 pages, 2019.

[14] S. K. Jo, H. R. Park, U. Jung, H. Oh, S. H. Kim, and S. T. Yee, "Protective effect of a herbal preparation (HemoHIM) on the self-renewal tissues and immune system against $\gamma$-irradiation," Journal of the Korean Society of Food Science and Nutrition, vol. 34, no. 6, pp. 805-813, 2005.

[15] H.-R. Park, S.-K. Jo, U.-H. Jung, S.-H. Kim, and S.-T. Yee, "Immunomodulatory effect of a new herbal preparation (HemoHIM) in cyclophosphamide-treated mice," Preventive Nutrition and Food Science, vol. 11, no. 1, pp. 54-60, 2006.

[16] N.-R. Shin, S.-H. Kim, J.-W. Ko et al., "HemoHIM, a herbal preparation, alleviates airway inflammation caused by cigarette smoke and lipopolysaccharide," Laboratory Animal Research, vol. 33, no. 1, pp. 40-47, 2017.

[17] D.-A. Kwon, Y. S. Kim, S. H. Baek et al., "Protective effects of a standardized extract (HemoHIM) using indomethacin- and ethanol/HCl-induced gastric mucosal injury models," Pharmaceutical Biology, vol. 57, no. 1, pp. 543-549, 2019.

[18] D. H. Kim, D. Y. Kim, Y. C. Kim et al., "Nodakenin, a coumarin compound, ameliorates scopolamine-induced memory disruption in mice," Life Sciences, vol. 80, no. 21, pp. 1944-1950, 2007.

[19] M. Yu, X. Chen, J. Liu et al., "Gallic acid disruption of A $\beta 1-42$ aggregation rescues cognitive decline of APP/PS1 double transgenic mouse," Neurobiology of Disease, vol. 124, pp. 67-80, 2019.

[20] M. Deng, H. Sun, J. Shen, Y. Fan, L. Zhang, and J. Zhang, "Radix angelica sinensis promotes synaptic plasticity during cognitive recovery in chronically stressed rats," Current Neurovascular Research, vol. 12, no. 3, pp. 232-239, 2015.

[21] S. H. Kwon, H. K. Lee, J. A. Kim et al., "Neuroprotective effects of chlorogenic acid on scopolamine-induced amnesia via antiacetylcholinesterase and anti-oxidative activities in mice," European Journal of Pharmacology, vol. 649, no. 1-3, pp. 210-217, 2010.

[22] M. Kato, R. Ochiai, K. Kozuma, H. Sato, and Y. Katsuragi, "Effect of chlorogenic acid intake on cognitive function in the elderly: a pilot study," Evidence-Based Complementary and Alternative Medicine, vol. 2018, Article ID 8608497, 8 pages, 2018.

[23] E. Heitman and D. K. Ingram, "Cognitive and neuroprotective effects of chlorogenic acid," Nutritional Neuroscience, vol. 20, no. 1, pp. 32-39, 2017.
[24] E. D. Levin, J. E. Rose, S. R. McGurk, and L. L. Butcher, "Characterization of the cognitive effects of combined muscarinic and nicotinic blockade," Behavioral and Neural Biology, vol. 53, no. 1, pp. 103-112, 1990.

[25] S. Granon, B. Poucet, C. Thinus-Blanc, J.-P. Changeux, and C. Vidal, "Nicotinic and muscarinic receptors in the rat prefrontal cortex: differential roles in working memory, response selection and effortful processing," Psychopharmacology, vol. 119, no. 2, pp. 139-144, 1995.

[26] T. Ahmed and A.-H. Gilani, "Inhibitory effect of curcuminoids on acetylcholinesterase activity and attenuation of scopolamine-induced amnesia may explain medicinal use of turmeric in Alzheimer's disease," Pharmacology Biochemistry and Behavior, vol. 91, no. 4, pp. 554-559, 2009.

[27] M. S. Kim, D. Y. Lee, J. Lee et al., "Terminalia chebula extract prevents scopolamine-induced amnesia via cholinergic modulation and anti-oxidative effects in mice," BMC Complementary and Alternative Medicine, vol. 18, no. 1, p. 136, 2018.

[28] M. Sarter, G. Bodewitz, and D. N. Stephens, "Attenuation of scopolamine-induced impairment of spontaneous alternation behaviour by antagonist but not inverse agonist and agonist ?-carbolines," Psychopharmacology, vol. 94, no. 4, pp. 491-495, 1988.

[29] H. K. Oh, S. J. Park, S. G. Bae et al., "Kami-ondam-tang, a traditional herbal prescription, attenuates the prepulse inhibition deficits and cognitive impairments induced by MK- 801 in mice," Journal of Ethnopharmacology, vol. 146, no. 2, pp. 600-607, 2013.

[30] K. J. Min, K. D. Hyun, P. S. Jin, and R. J. Hoon, "Memory enhancing properties of the ethanolic extract of black sesame and its ameliorating properties on memory impairments in mice," Korean Journal of Pharmacognosy, vol. 41, no. 3, pp. 196-203, 2010.

[31] I. Klinkenberg and A. Blokland, "The validity of scopolamine as a pharmacological model for cognitive impairment: a review of animal behavioral studies," Neuroscience \& Biobehavioral Reviews, vol. 34, no. 8, pp. 1307-1350, 2010.

[32] A. T. Mugwagwa, L. L. Gadaga, W. Pote, and D. Tagwireyi, "Antiamnesic effects of a hydroethanolic extract of Crinum macowanii on scopolamine-induced memory impairment in mice," Journal of Neurodegenerative Diseases, vol. 2015, Article ID 242505, 9 pages, 2015.

[33] R. Agrawal, E. Tyagi, G. Saxena, and C. Nath, "Cholinergic influence on memory stages: a study on scopolamine amnesic mice," Indian Journal of Pharmacology, vol. 41, no. 4, p. 192, 2009.

[34] M. D. Pandareesh, T. Anand, and F. Khanum, "Cognition enhancing and neuromodulatory propensity of Bacopa monniera extract against scopolamine induced cognitive impairments in rat hippocampus," Neurochemical Research, vol. 41, no. 5, pp. 985-999, 2016.

[35] M. Wong-Guerra, J. Jiménez-Martin, L. A. Fonseca-Fonseca et al., "JM-20 protects memory acquisition and consolidation on scopolamine model of cognitive impairment," Neurological Research, vol. 41, no. 5, pp. 385-398, 2019.

[36] T. Myhrer, "Neurotransmitter systems involved in learning and memory in the rat: a meta-analysis based on studies of four behavioral tasks," Brain Research Reviews, vol. 41, no. 2-3, pp. 268-287, 2003.

[37] P. Palit, D. Mukherjee, and S. C. Mandal, "Reconstituted mother tinctures of Gelsemium sempervirens L. improve memory and cognitive impairment in mice scopolamineinduced dementia model," Journal of Ethnopharmacology, vol. 159, pp. 274-284, 2015. 
[38] S. A. Rosales-Corral, D. Acuña-Castroviejo, A. Coto-Montes et al., "Alzheimer's disease: pathological mechanisms and the beneficial role of melatonin," Journal of Pineal Research, vol. 52, no. 2, pp. 167-202, 2012.

[39] J. L. Hoskin, Y. Al-Hasan, and M. N. Sabbagh, "Nicotinic acetylcholine receptor agonists for the treatment of Alzheimer's dementia: an update," Nicotine \& Tobacco Research, vol. 21, no. 3, pp. 370-376, 2019.

[40] M. S. García-Ayllón, I. Riba-Llena, C. Serra-Basante, J. Alom, R. Boopathy, and J. Sáez-Valero, "Altered levels of acetylcholinesterase in Alzheimer plasma," PLoS One, vol. 5, no. 1, 2010.

[41] I. C. Jung, S. R. Lee, and S. H. Yun, "Effects of Cyperus rotundus (CPRT) on inhibition of impairment of learning and memory, and acetylcholinesterase in amnesia mice," Journal of Oriental Neuropsychiatry, vol. 14, no. 1, pp. 59-74, 2003.

[42] L. X. Zhao, Y. H. Ge, C. H. Xiong et al., "M1 muscarinic receptor facilitates cognitive function by interplay with AMPA receptor GluAl subunit," The FASEB Journal, vol. 32, no. 8, pp. 4247-4257, 2018.

[43] M. Ishii and Y. Kurachi, "Muscarinic acetylcholine receptors," Current Pharmaceutical Design, vol. 12, no. 28, pp. 3573-3581, 2006.

[44] P. Kása, Z. Rakonczay, and K. Gulya, "The cholinergic system in Alzheimer's disease," Progress in Neurobiology, vol. 52, no. 6, pp. 511-535, 1997.

[45] B. H. Chen, J. H. Park, T.-K. Lee et al., "Melatonin attenuates scopolamine-induced cognitive impairment via protecting against demyelination through BDNF-TrkB signaling in the mouse dentate gyrus," Chemico-biological Interactions, vol. 285, pp. 8-13, 2018.

[46] Q. Wang, F. Shao, and W. Wang, "Region-dependent alterations in cognitive function and ERK1/2 signaling in the PFC in rats after social defeat stress," Neural Plasticity, vol. 2018, Article ID 9870985, 11 pages, 2018.

[47] P. Kowiański, G. Lietzau, E. Czuba, M. Waśkow, A. Steliga, and J. Moryś, "BDNF: a key factor with multipotent impact on brain signaling and synaptic plasticity," Cellular and Molecular Neurobiology, vol. 38, no. 3, pp. 579-593, 2018. 